\title{
Higher alpha-synuclein aggregate density does not lead to more severe dopaminergic cell loss in the AAV-mediated overexpression model of Parkinson's Disease: A time- course study
}

\author{
Banu Cahide TEL ${ }^{1}$ * (D) İnci KAZKAYASI ${ }^{1} \mathbb{D}$, Gökçen TELLİ ${ }^{1}(\mathbb{D})$, Elif ÇINAR ${ }^{2} \mathbb{( D )}$, \\ Sevgi Uğur MUTLUAY ${ }^{1}\left(\mathbb{D}\right.$, Gül YALÇIN-ÇAKMAKLI ${ }^{3}{ }^{(D)}$, Esen SAKA ${ }^{3}$ (D) , Bülent ELİBOL ${ }^{3}$ (D) \\ 1 Department of Pharmacology, Faculty of Pharmacy, Hacettepe University, Ankara, Turkey. \\ 2 Department of Pharmacology, Faculty of Pharmacy, Zonguldak Bülent Ecevit University, Zonguldak, Turkey. \\ 3 Department of Neurology, Faculty of Medicine, Hacettepe University, Ankara, Turkey. \\ * Corresponding Author. E-mail: banutel@hacettepe.edu.tr (B.C.T.); Tel. +90-312-305 2131.
}

Received: 25 March 2021/ Revised: 04 May 2021/ Accepted: 07 May 2021

ABSTRACT: Pathological intracellular aggregation of alpha-synuclein (a-syn) is the hallmark of Parkinson's disease (PD). Our aim is to explore the outcomes of long-term a-syn pathology with its functional correlates in the PD model by AAV (adeno-associated virus)-mediated a-syn overexpression in substantia nigra (SN). Female Wistar rats (220-260 g) received a unilateral injection of AAV-human-a-syn or green fluorescent protein (GFP) gene into the SN. The animals were tested for motor functions with cylinder test at 8, 12 weeks or 9 months post-injection. The intensity of a-syn accumulation or GFP in striatum and dopaminergic neuronal loss in $\mathrm{SN}$, dopaminergic terminal loss in striatum and synaptic integrity were analyzed by a-syn, GFP, tyrosine hydroxylase (TH) and synaptophsin immunohistochemistry, respectively. At all time-points, AAV-human-a-syn injected animals displayed more motor dysfunction and THpositive cell loss compared to AAV-GFP injected group. A-syn immunoreactivity was present in the nigral neurons as well as the striatal terminals in all animals that received AAV-a-syn. Striatal TH density analysis showed a decrease in both 12-weeks and 9 months a-syn groups compared to controls. However, TH-positive neuron count was lower in 9months group compared to 12-weeks group. Hence, the motor performance of 9-month group showed an improvement which may be a sign of a compensatory mechanisms against a-syn-induced neurodegeneration. The findings of this study implicate that higher a-syn density in SN does not always lead to worse motor function or more severe dopaminergic cell loss. This may support the hypothesis that a-syn aggregates are the end-product of a cellular defense mechanism rather than being causative pathology.

KEYWORDS: Alpha-synuclein; Parkinson's disease; adeno associated viral vectors; animal model.

\section{INTRODUCTION}

Parkinson's disease (PD) is the second most common neurodegenerative disease characterized by progressive death of neurons in substantia nigra pars compacta (SNpc). Although the reason of the progressive nature of nigral cell death is still unclear, Lewy bodies are thought to be closely related to the pathology. Alpha-synuclein (a-syn) is an abundant synaptic protein which was identified as the major component of the Lewy bodies. It plays a role in vesicular trafficking, protein phosphorylation, signal transduction, maintaining mitochondrial function, and many more cellular pathways and it may also interact with the protein degradation pathways. It may induce oxidative stress, and/or mitochondrial dysfunction and even cell death. However, it is still controversial that the aggregation process is whether responsible from the neurodegenerative process or is just an end-product of a cellular defense mechanism [1].

Although PD is mainly characterized by motor symptoms, the pathological process starts a lot earlier in the central (olfactory nucleus) and peripheral nervous system (enteric plexus). It progresses to the rostral brain regions, resulting in non-motor symptoms (NMS), which can generally be classified as neuropsychiatric, gastrointestinal, autonomic, sensory, and sleep disorders. Presenting long before the motor symptoms and progressing during the course of the disease, non-motor symptoms affect the quality of life as much as the

How to cite this article: Tel BC, Kazkayasi I, Telli G, Cinar E, Mutluay SU, Yalcin-Cakmakli G, Saka E, Elibol B. Higher alpha-synuclein aggregate density does not lead to more severe dopaminergic cell loss in the AAV-mediated overexpression model of Parkinson's Disease: A time-course study. J Res Pharm. 2021; 25(3): 352-358. 
motor symptoms. Current understandings of pathological correlates of NMS is rapidly increasing alongside with the recent interest to these symptoms in clinical practice. Likewise, various animal models trying to recapitulate NMS are increasing in number in order to characterize the timing and development of pathology, which is essential to recognize target pathways for therapeutic interventions $[2,3]$.

Toxin-mediated PD animal models have long been used to study late-stage PD or to test dopaminergic agents in the backdrop of severe dopaminergic neuronal loss in SN. However, in these models neither a-syn aggregation, the pathological hallmark of the disease, can be induced, nor the progressive nature of the neurodegenerative process. Therefore, toxin-mediated models have been rapidly replaced by alphasynucleinopathy models such as transgenic, viral vector-mediated and recombinant a-syn fibril models. These models provided the opportunity to study the pathogenetic processes centered on a-syn aggregation with a better mechanistic insight.

Viral vectors are powerful tools to deliver a specific gene in the desired brain region. Previously, adenoassociated viral vectors were utilized to overexpress a-syn in SN in rodent models successfully with progressive death of nigral dopaminergic neurons and alpha-synuclein inclusions. Motor signs have also been recapitulated in this model [4-9]. However, the dopaminergic neuronal loss was found around $50 \%$ [10], the motor dysfunction manifested in adeno- associated virus (AAV)-mediated a-syn overexpression model reflects only as mild Parkinsonism. Since the accumulation of a-syn increase in time, later time-points in AAVmediated a-syn overexpression model may be expected to lead to more severe motor symptoms, however, this has not been investigated yet. In this study, we aimed to explore the outcomes of long-term a-syn pathology with its functional correlates in the PD model of AAV-mediated a-syn overexpression in SN.

\section{RESULTS}

In cylinder test, naïve animals did not show any bias towards left or right paw (Figure 1). There was no difference in the ratio of the contralateral paw contacts to total contacts in a-syn or GFP groups compared to the naïve controls at $8^{\text {th }}$ weeks. At $12^{\text {th }}$ week although the contralateral contact decreased in a-syn group, it did not reach statistical significance. Similar pattern was observed in 12-week GFP group. However, in 9-month group the motor asymmetry recovered hence the number of the contralateral contacts increased back to control level both in a-syn and GFP injected groups (Figure 1).

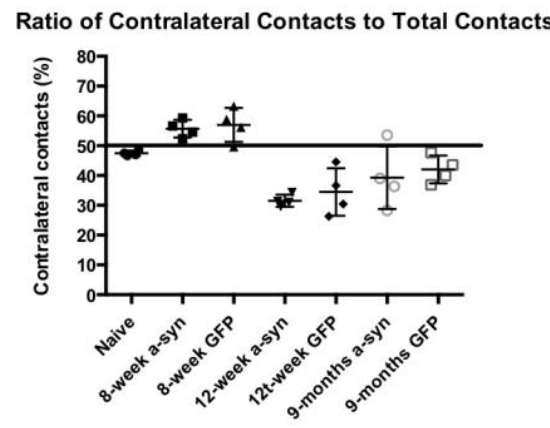

Figure 1. Results of motor tests: In cyclinder test; contralateral forelimb use decrease in 12-week a-syn injected animals was not significant compared to the control. However, at 9 months, the contralateral forelimb use was increased compared to 12-week group ( $>0.05)$. a-syn: Alpha-synuclein, GFP: Green Floresence Protein ( $n=4,8$-week a-syn; n=4, 8-week GFP; n=4, 12-week a-syn; n=4, 12-week GFP; n=4, 9month a-syn; n=4, 9-month GFP; n=4, naïve control).

Immunohistochemistry (IHC) analysis revealed a distinct pattern of a-syn overexpression as previously reported [11]. A-syn labelling in SN and striatum was widespread and observed only ipsilateral to the side in all groups except in 9-month group, in which the expression was also observed in the contralateral side of the midbrain (Figure 2D). In detailed analysis, a-syn accumulation was detected in cell bodies and dendritic processes in SN and synaptic terminals in the striatum (Figure 2E).

AAV-mediated a-syn and GFP overexpression became prominent at the end of the $6^{\text {th }}$ week in $\mathrm{SN}$ and its projection area, striatum (Figure 2A, 2F). The overexpression of a-syn and GFP increased in time in both $\mathrm{SN}$ and striatum (Figure 2A-I). The a-syn aggregation in $\mathrm{SN}$ was the lowest at $8^{\text {th }}$ week post-injection when compared between three time-points. $6^{\text {th }}$ week groups are not included in semi-quantitave analysis due to very low level of transgene expression. More robust a-syn overexpression was detected in $\mathrm{SN}$ of the 9-month group compared to the 12-week group (Figure 3A, 2B-D). 


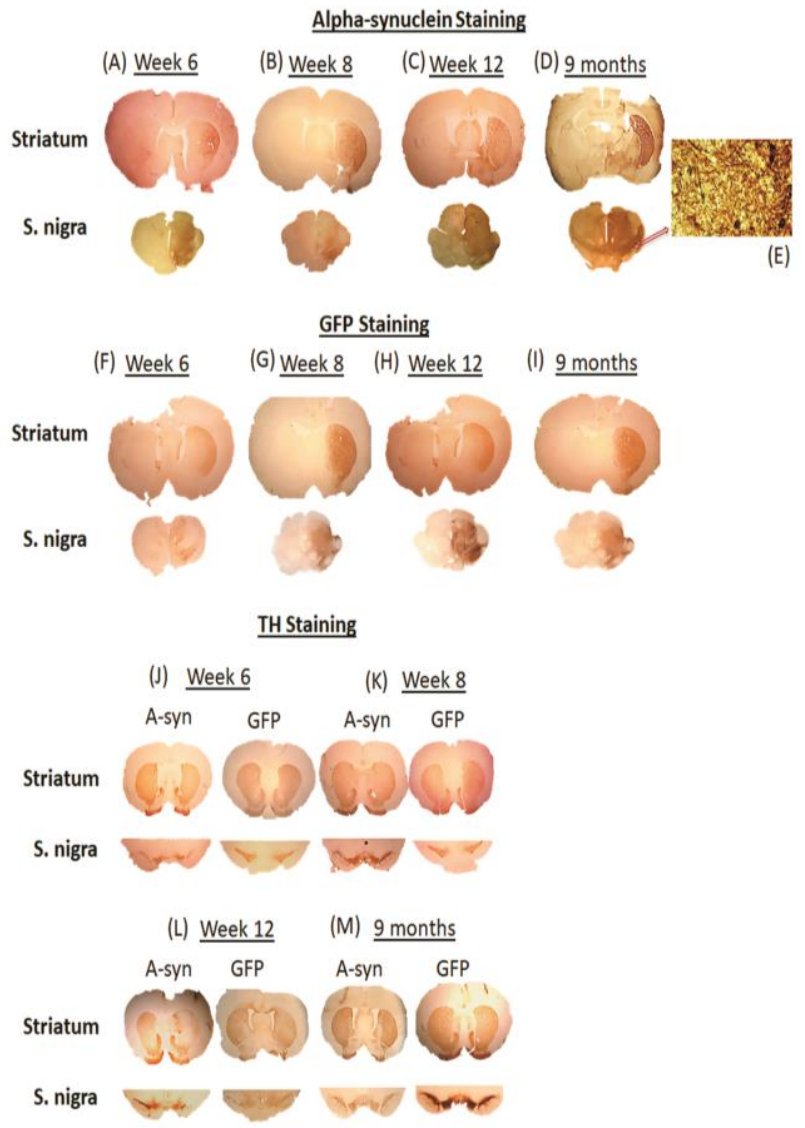

Figure 2. Representative pictures from immunohistochemical staining of alpha-synuclein (A-D), green fluorescent protein (GFP) (F-I), and TH (J-M) staining in a-syn or GFP injected groups at 6-week, 8-week, 12-week and 9-month time-points ( $\mathrm{n}=4,6$-week a-syn; n=4, GFP; n=4, 8-week a-syn; n=4, 8-week GFP; n=4, 8-week a-syn; n=4, 8-week GFP; n=4, 12-week a-syn; n=4, 12-week GFP; n=4, 9-month a-syn; n=4, 9-month $\mathrm{GFP} ; \mathrm{a}-\mathrm{syn}=$ alpha-synuclein, $\mathrm{GFP}=$ green fluorescent protein, $\mathrm{TH}=$ tyrosine hydroxylase) .

In order to determine the a-syn-related neuronal loss, nigral and striatal sections from $6^{\text {th }}, 8^{\text {th }}, 12^{\text {th }}$ week and $9^{\text {th }}$ month animals were stained with anti-TH antibody (Figure 2J-M). Similar to the motor asymmetry results, increased $\mathrm{TH}$-positive $\left(\mathrm{TH}^{+}\right)$-neuronal loss in $\mathrm{SN}$ as well as decreased $\mathrm{TH}$ density in striatum in 12week group was detected. However, the $\mathrm{TH}^{+}$-neuronal loss and $\mathrm{TH}$ density levels were found similar to the control levels in 9-month group (Figure 3B-C, $\mathrm{p}<0.02$ and $\mathrm{p}<0.02$ ).

To evaluate the synaptic integrity at $9^{\text {th }}$ month, synaptophysin immunohistochemical staining in SN was analyzed by ImageJ and in striatum of a-syn or GFP injected animals. There was no difference in the nigral synaptophysin density in a-syn groups compared to naïve controls (Figure 3D). The striatal synaptophysin density was higher than that of naïve control group although this did not reach statistical significance (Figure 3E).

\section{DISCUSSION}

The primary goal of the present study was to establish an in vivo model that recapitulates the progressive nature of a-syn pathology. For this reason, we chose AAV vectors to induce the a-syn overexpression in later time-points and conducted cylinder test to follow the development of motor phenotype. Furthermore, we assessed whether the pathological changes correlated with the motor impairment. It was shown that a-syn and GFP were successfully expressed in the targeted areas by IHC. A-syn and GFP expression were also observed in the striatum, which is the projection area of the $\mathrm{SN}$ dopaminergic neurons. The transgene overexpressions occurred in a time-dependent manner. These results are in accordance with the previous studies showing that AAV serotype 6 led to an efficient transduction both in the target and projection areas in the brain $[4,5]$. 
A
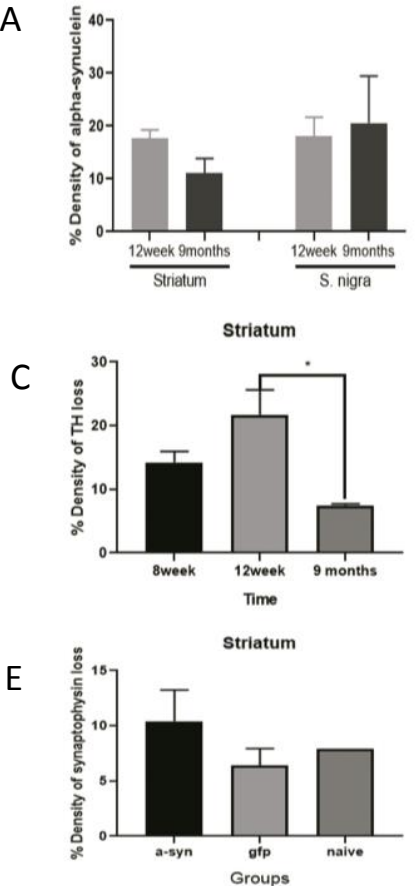
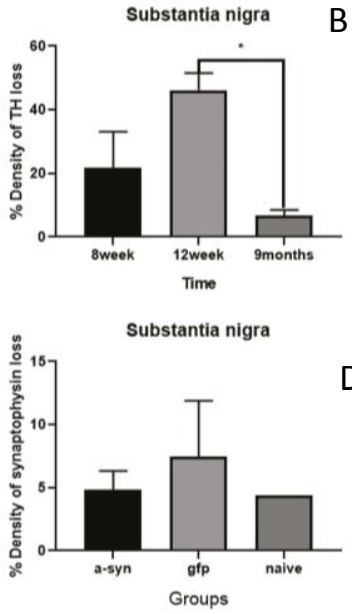

Figure 3. Results of densitometric analysis. (A) Quantitative analysis of a-syn accumulation as a \% of a-syn density in SN and striatum compared to intact side by ImageJ (B) Semi-quantitative analysis of TH staining in $\mathrm{SN}$; the TH+-neuronal loss was increased in 12-week group, but this loss was reversed at 9 months group ( $p<0.02,12$-week a-syn vs 9-month). (C) Semi-quantitative analysis of TH density in the striatum. The \% of TH density in striatum compared to intact side by ImageJ. The TH density increased in 12-week group, but this increase was reversed at 9 months group ( $p<0.02,12$-week a-syn vs 9 -month). (D) Semi-quantitative analysis of AAV-a-syn injection to $\mathrm{SN}$ did not cause a decrease in synaptophysin levels compared to naïve animals or (E) in striatum after 9 months $(\mathrm{p}>0.05)$. S. nigra: Substantia nigra, a-syn: Alpha-synuclein, TH: Tyrosine hydroxylase ( $n=4,8$-week a-syn; n=4, 8-week GFP; n=4, 12-week a-syn; n=4, 12-week GFP; n=4, 9month a-syn; n=4, 9-month GFP; $n=4$, naïve control).

Cylinder test was performed to assess motor asymmetry. There was a decrease in the contralateral forelimb use only at $12^{\text {th }}$ week time-point compared to controls. The $8^{\text {th }}$ week group did not show any forelimb bias indicating that there was no motor asymmetry, which is a result of insufficient striatal dopaminergic denervation $[5,6]$. However, at $9^{\text {th }}$ month time-point, the animals showed better motor performance than 12week group which suggests that there had been a recovery or compensation mechanism for neuronal survival due to long term exposure the protein aggregates. There is a mild asymmetry found in GFP-injected group. SN might be selectively susceptible to potential toxicity of AAV-mediated GFP expression, as was suggested before $[7,8,9]$, therefore, GFP toxicity may occurred in the titration we used for our experiments.

TH immunostaining was performed to analyze the impact of a-syn accumulation on neuronal viability. The estimation of $\mathrm{TH}^{+}$-staining neurons was counted in the SNpc. In addition to that, $\mathrm{TH}$ density was measured by semi-quantitative image analysis in the striatum. There was a mild but marked decrease in $\mathrm{TH}^{+}-$ staining neurons in a-syn overexpressing animals both in 8- and 12-week groups, respectively. This $\mathrm{TH}^{+}-$ neuronal loss was reflected also in the striatal TH density both in 8- and 12-week groups. These findings are in accordance with the previous results [11, 15]. However, there is a marked recovery observed in 9-month group, this might be as a result of TH enzyme loss rather than complete loss of the dopaminergic neurons themselves. In a study of Albert et al, the loss of TH phenotype phenomenon in dopaminergic neurons was described. There was an inconsistency with the decreased amount of $\mathrm{TH}^{+}$-neurons compared to motor deficit [16]. However, the $\mathrm{TH}^{+}$-neuronal loss and motor asymmetry was found to be parallel in our findings, which may indicate that there may be another mechanism involved other than loosing TH phenotype. In addition to our earlier findings, here we show compensatory motor mechanisms may play a role against a-syn induced neurodegeneration

In order to evaluate the synaptic loss, we then measured the striatal synaptophysin density in all animal groups. IHC analysis revealed that a-syn overexpression did not lead to a decrease in synapthophysin density at any time point (only the 9 months shown). On the other hand, in striatum synaptophysin density showed a trend to increase in 9-month a-syn group. Interestingly, regardless of significance levels, this increase indicates 
that there might be a synaptic compensation occurring in the striatum, which could also explain the better motor performance of 9-month group.

The results of the current study showed that a-syn accumulation increased in time but after a certain point, even though the accumulation continued to increase, the pathological process leading to dopaminergic neuronal loss and motor asymmetry halted, seemed to reach a limit. There are some studies, which investigated a-syn overexpression beyond 12 weeks $[17,18]$. However there is a one-year study in primates with a different serotype viral vector carrying wild-type a-syn [18], similarly, the motor deficits diminished after 27 weeks and they found only mild nigral dopaminergic neuronal loss in post-mortem analysis.

This is the first study, which investigates the clinicopathological correlates of AAV-mediated a-syn overexpression PD model in rats time course-wise. This model can be useful for studying the alpha-synuclein related pathology in idiopathic $\mathrm{PD}$ as well as helpful to guide the researchers for choosing the best time-point when studying in AAV-mediated a-syn overexpression PD model in rat. According to our results the optimum time point would be around 12 weeks.

\section{CONCLUSION}

In conclusion, the findings of this study implicate that higher a-syn aggregate density does not always lead to worse motor function or more severe dopaminergic cell loss. This may support the hypothesis that asyn aggregates are the end-product of a cellular defense mechanism rather than being the causative pathology.

\section{MATERIALS AND METHODS}

\subsection{Animals}

Female Wistar rats (aged 10-12 weeks, weighing 220-260 g) were used (n=36). Rats were brought to the laboratory at least one week before the experiments and kept under standard conditions with ad libitum access to food and water. All experimental procedures were approved by Hacettepe University Local Animal Ethics Committee (Decision number: 2014/44-10) and performed in compliance with national and local animal care and use guidelines.

Animals were divided into two groups and injected with AAV-carrying human wild-type a-syn $(\mathrm{n}=16)$ or green fluorescent protein (GFP, n=16) into SN unilaterally. Further, 4 animals were used as naïve controls. Each group was divided into four groups; histopathological evaluations were performed at $6^{\text {th }}, 8^{\text {th }}, 12^{\text {th }}$ or $9^{\text {th }}$ month ( $36^{\text {th }}$ week) post-injection for following up the course of overexpression at different time-points. Animals were tested for motor changes only at $8^{\text {th }}, 12^{\text {th }}$ or $9^{\text {th }}$ month weeks after injection. At the end of each time-point animals were sacrificed, and brain sections were analyzed by IHC.

\subsection{Surgery}

Animals were deeply anesthetized with a mix of ketamine and xylazine and placed in a stereotaxic frame (Stoelting, USA). The toothbar was set to $-2.3 \mathrm{~mm}$ [19]. Coordinates for SN injections were anteroposterior $(\mathrm{AP})=-5.2 \mathrm{~mm}$ and mediolateral $(\mathrm{ML})=-2.0 \mathrm{~mm}$ relative to bregma and dorsoventral $(\mathrm{DV})=-$ $7.2 \mathrm{~mm}$ from dura. The injections were made by a $5 \mu \mathrm{l}$ Hamilton syringe fitted with a pulled glass capillary. Viral vectors were purchased from Prof. Deniz Kirik's laboratory (BRAINS Unit, Lund University). $2 \mu 1$ of vector suspension containing $\mathrm{rAAV6-a-syn}(5.9 \mathrm{E} 13 \mathrm{vg} / \mathrm{ml})$ or $r A A V 6-G F P(6.9 E 13 \mathrm{vg} / \mathrm{ml})$ was injected slowly at a rate of $0.2 \mu \mathrm{l} /$ minute as described previously [11,12].

\subsection{Cylinder test (CT)}

For evaluating motor asymmetry due to unilateral injection, CT was performed. Number of contralateral forelimb contacts on the cylinder wall was analyzed. A glass cylinder $25 \mathrm{~cm}$ in diameter and three mirrors to see touches from all angles were used. The room was kept dim. Rats were put in the cylinder and recorded for ten minutes with a video camera (Sony Handycam DCR-HC37). A blinded and trained observer analyzed recordings. The contralateral contacts were expressed as the percentage of total number (ipsilateral+contralateral) of contacts. 


\subsection{Immunohistochemistry (IHC)}

Animals were perfused transcardially with heparine followed by $4 \%$ paraformaldehyde infusion under terminal anesthesia. Brains were cut into $35 \mu \mathrm{m}$-thick coronal slices with microtome (Leica SM2000 R, Leica Biosystems). Immunohistochemical staining was performed with Acu-Stain HRP Kit (52-0003, Genemed) on free-floating slices. All steps were carried out at room temperature as described earlier [10]. In brief, slices were rinsed with Tris- $\mathrm{HCl}$ solution then quenched for 20 minutes and rinsed again with Tris-buffered saline solution. Then they were pre-incubated with blocking solution from the kit for 10 minutes. After blocking, slices were incubated with monoclonal anti-a-syn (1:1000, ab80627, Abcam), anti-GFP (1:1000, ab13970, Abcam), anti-TH (1:1000, ab75875, Abcam) or anti-synaptophsin (S-5768, Sigma-Aldrich) antibodies overnight, rinsed 3 times and incubated with biotinylated secondary antibody solution from the kit for 10 minutes. After rinse, slices were incubated with Streptavidin-peroxidase complex for 10 minutes and rinsed for the last time, then they were developed by 3,3-diaminobenzidine as a chromogen and $\mathrm{H}_{2} \mathrm{O}_{2}$ as a catalyst (Power- StainTM 1.0 Poly HRP DAB Kit, Genemed), mounted on slides and cover slipped with DPX mounting medium.

\subsection{Density measurement}

Relative intensity of striatal alpha-synuclein and synaptophysin immunostaining was assessed on stained tissue slides containing different levels of striatum and SN. ImageJ [20] was used for optical density measurement. The images were converted into 16-bit grayscale format and inverted. To eliminate the background staining, the optical density measurement in the corpus callosum was subtracted from that obtained in striatal measurements. Intensity measurements were performed on the injection side of different sections from 3 adjacent sections at rostro-caudal levels and obtained a mean value for each animal. In each striatal section, the intensity was measured in the dorsolateral part of striatum. The average intensity values were calculated for each experimental group for comparison.

For the nigral TH neuronal count, TH-immunostained sections containing different levels of SNpc were photographed under upright brightfield microscope (Nikon Eclipse E600, Tokyo, Japan) attached with a digital color camera (Nikon, Tokyo, Japan). Regions of interest were delineated on these photographs and number of TH+-neuronal bodies were counted by two blind observers manually. Photographs were taken at a $20 \times 3.3$ magnification per area with a standardized illumination to distinguish background staining [11].

\subsection{Statistical analysis}

Data were presented as mean \pm standard error of mean. The significance threshold was set at 0.05 . Twotail unpaired t-test was used. All data were analyzed in GraphPad PRISM v6.0 software.

Acknowledgements: This study was supported by Hacettepe University Scientific Research Projects Coordination Unit (014 D08 301 007-701). We would like to thank Dr. Ayse Ulusoy for her invaluable contributions in developing and establishing the AAV-associated alpha-synuclein rat model. We would like to thank to all of the members of Hacettepe University Institute of Neurological Sciences and Psychiatry Brain Research Laboratory for their support and help to use all the equipment's in the lab.We also would like to thank Necati Şengönül, Erdoğan Aksay and Mesut Frrat for their technical support.

Author contributions: Concept - B.C.T., B.E.; Design - B.C.T, E.S., B.E.; Supervision - B.C.T., G.Y.Ç.; Resources - B.C.T., E.S., B.E..; Materials - İ.K., G.T., E.Ç., S.U.M.; Data Collection and/or Processing - G.Y-Ç., İ.K., G.T., E.Ç., S.U.M; Analysis and/or Interpretation - B.C.T, G.Y.-Ç., E.Ç..; Literature Search - B.C.T.; Writing - B.C.T.; Critical Reviews B.C.T., İ.K., G.T., E.Ç., S.U.M., G.Y.Ç., E.S., B.E.

Conflict of interest statement: The authors declared no conflict of interest.

Ethics committee approval: All experimental procedures were approved by Hacettepe University Local Animal Ethics Committee (Decision number: 2014/44-10) and performed in compliance with national and local animal care and use guidelines.

\section{REFERENCES}

[1] Chartier S, Duyckaerts C. Is Lewy pathology in the human nervous system chiefly an indicator of neuronal protection or of toxicity? Cell Tissue Res. 2018; 373(1): 149-60. [CrossRef]

[2] Titova N, Schapira AHV, Chaudhuri KR, Qamar MA, Katunina E, Jenner P. Nonmotor Symptoms in Experimental Models of Parkinson's Disease. Int Rev Neurobiol. 2017; 133: 63-89. [CrossRef] 
[3] Chiu WH, Depboylu C, Hermanns G, Maurer L, Windolph A, Oertel WH, Ries V, Höglinger GU. Long-term treatment with L-DOPA or pramipexole affects adult neurogenesis and corresponding non-motor behavior in a mouse model of Parkinson's disease. Neuropharm. 2015; 95: 367-76. [CrossRef]

[4] Kirik D, Rosenblad C, Burger C, Lundberg C, Johansen TE, Muzyczka N, et al. Parkinson-like neurodegeneration induced by targeted overexpression of alpha-synuclein in the nigrostriatal system. J Neurosci. 2002; 22(7): $2780-91$. [CrossRef]

[5] Decressac M, Mattsson B, Lundblad M, Weikop P, Bjorklund A. Progressive neurodegenerative and behavioural changes induced by AAV-mediated overexpression of alpha-synuclein in midbrain dopamine neurons. Neurobiol Dis. 2012; 45(3): 939-53. [CrossRef]

[6] Gombash SE, Manfredsson FP, Kemp CJ, Kuhn NC, Fleming SM, Egan AE, et al. Morphological and behavioral impact of AAV2/5-mediated overexpression of human wildtype alpha-synuclein in the rat nigrostriatal system. PLoS One. 2013; 8(11): e81426. [CrossRef]

[7] Oliveras-Salva M, Van der Perren A, Casadei N, Stroobants S, Nuber S, D'Hooge R, et al. rAAV2/7 vector-mediated overexpression of alpha-synuclein in mouse substantia nigra induces protein aggregation and progressive dosedependent neurodegeneration. Mol Neurodegener. 2013; 8: 44. [CrossRef]

[8] Van der Perren A, Van den Haute C, Baekelandt V. Viral vector-based models of Parkinson's disease. Curr Top Behav Neurosci. 2015; 22: 271-301. [CrossRef]

[9] Bourdenx M, Dovero S, Engeln M, Bido S, Bastide MF, Dutheil N, et al. Lack of additive role of ageing in nigrostriatal neurodegeneration triggered by alpha-synuclein overexpression. Acta Neuropathol Commun. 2015; 3: 46. [CrossRef]

[10] Ulusoy A, Decressac M, Kirik D, Bjorklund A. Viral vector-mediated overexpression of alpha-synuclein as a progressive model of Parkinson's disease. Prog Brain Res. 2010; 184: 89-111. [CrossRef]

[11] Mutluay SU, Çınar E, Yalçın Çakmaklı G, Ulusoy A, Elibol B, Tel BC. Modelling Non-motor Symptoms of Parkinson's Disease: AAV Mediated Overexpression of Alpha-synuclein in Rat Hippocampus and Basal Ganglia. Turk J Neurol. 2020; 26(4): 322-329. [CrossRef]

[12] Albert K, Voutilainen MH, Domanskyi A, Airavaara M. AAV Vector-Mediated Gene Delivery to Substantia Nigra Dopamine Neurons: Implications for Gene Therapy and Disease Models. Genes (Basel). 2017; 8(2): 63. [CrossRef]

[13] Koprich JB, Johnston TH, Huot P, Reyes MG, Espinosa M, Brotchie JM. Progressive neurodegeneration or endogenous compensation in an animal model of Parkinson's disease produced by decreasing doses of alphasynuclein. PLoS One. 2011; 6(3): e17698. [CrossRef]

[14] Landeck N, Buck K, Kirik D. Toxic effects of human and rodent variants of alpha-synuclein in vivo. Eur J Neurosci. 2017; 45(4): 536-547. [CrossRef]

[15] Cinar E, Yalcin-Cakmakli G, Saka E, Ulusoy A, Yuruker S, Elibol B, et al. Modelling cognitive deficits in Parkinson's disease: Is CA2 a gateway for hippocampal synucleinopathy? Exp Neurol. 2020; 330: 113357. [CrossRef]

[16] Albert K, Voutilainen MH, Domanskyi A, Piepponen TP, Ahola S, Tuominen RK, et al. Downregulation of tyrosine hydroxylase phenotype after AAV injection above substantia nigra: Caution in experimental models of Parkinson's disease. J Neurosci Res. 2019; 97(3): 346-61. [CrossRef]

[17] Delenclos M, Faroqi AH, Yue M, Kurti A, Castanedes-Casey M, Rousseau L, et al. Neonatal AAV delivery of alphasynuclein induces pathology in the adult mouse brain. Acta Neuropathol Commun. 2017; 5(1): 51. [CrossRef]

[18] Eslamboli A, Romero-Ramos M, Burger C, Bjorklund T, Muzyczka N, Mandel RJ, et al. Long-term consequences of human alpha-synuclein overexpression in the primate ventral midbrain. Brain. 2007; 130(Pt 3): 799-815. [CrossRef]

[19] Paxinos G WC. The Rat Brain in Stereotaxic Coordinates: Hard Cover Edition: AcademicPress; 2006.

[20] Schneider CA, Rasband WS, Eliceiri KW. NIH Image to ImageJ: 25 years of image analysis. Nat Methods. 2012; 9(7): 671-5. [CrossRef] 